\title{
Carnets
}

Revue électronique d'études françaises de l'APEF

Première Série - 5 | 2013

Métamorphoses littéraires

\section{Philomena de Chretien de Troyes: Métamorphose d'une métamorphose au temps du roman}

Sylvia Roustant

\section{OpenEdition}

Journals

\section{Édition électronique}

URL : http://journals.openedition.org/carnets/8240

DOI : 10.4000/carnets.8240

ISSN : 1646-7698

Éditeur

APEF

Édition imprimée

Date de publication : 1 mai 2013

Pagination : 63-76

Référence électronique

Sylvia Roustant, « Philomena de Chretien de Troyes: Métamorphose d'une métamorphose au temps du roman », Carnets [En ligne], Première Série - 5 | 2013, mis en ligne le 23 juin 2018, consulté le 20 avril 2019. URL : http://journals.openedition.org/carnets/8240; DOI : 10.4000/carnets.8240

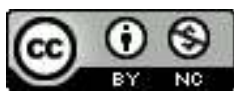

Carnets est mis à disposition selon les termes de la licence Creative Commons - Atribution - Pas d'utilisation commerciale 4.0 International. 


\title{
PHILOMENA DE CHRETIEN DE TROYES Métamorphose d'une métamorphose au temps du roman
}

\author{
SYLVIA ROUSTANT \\ Lycée Charles le Chauve de Roissy, en Brie \\ sroustant@free.fr
}

\begin{abstract}
Résumé
Cette œuvre de Chrétien de Troyes a souvent été lue, étudiée, au sein de L'Ovide moralisé (XIVe siècle) où elle a été découverte mais qui est postérieur à l'écriture de Philomena (fin XII ${ }^{\mathrm{e}}$ siècle). II s'agit donc de la replacer dans son contexte d'écriture: l'avènement du roman, genre nouveau utilisant une langue d'écriture nouvelle. Philomena est ainsi d'abord l'histoire d'une métamorphose linguistique, le latin jusqu'alors utilisé à l'écrit cédant la place à la langue romane. II s'agit également de replacer Philomena dans l'œuvre de Chrétien de Troyes, en montrant qu'elle contient en germe les principaux thèmes et questionnements des œuvres de Chrétien. Philomena est aussi l'histoire d'une métamorphose culturelle dans la mesure où l'auteur réécrit le mythe comme un contre-modèle de la courtoisie. Pour envisager Philomena comme un moment essentiel sur le plan linguistique et culturel, nous nous appuierons d'abord sur le traitement du temps, très différent chez Chrétien de Troyes.
\end{abstract}

\begin{abstract}
This work of Chrétien de Troyes has often been read, studied, within l'Ovide moralisé (fourteenth century), where it has been discovered, but which is posterior to its writing (late twelfth century). We therefore have to place this work in context: the advent of roman, new kind of genre using a new kind of writing language. Thus Philomena is primarily the story of a linguistic metamorphosis. Latin previously used in written works, is giving way to the Romance language. We also have to place Philomena among the other pieces of the work of Chrétien de Troyes, showing in particular it contains the germ of the main themes and questions of Chrétien's works. Philomena is also the story of a cultural metamorphosis since the author has rewritten this myth as a cons-model of courtesy. To consider Philomena as an essential moment in terms of language and culture, we will rely primarily on the handling of the time, really different in this work of Chrétien de Troyes.
\end{abstract}

Mots-clés: Chrétien de Troyes - Philomena - roman - métamorphose - Ovide

Keywords: Chrétien de Troyes - Philomena - roman - metamorphosis - Ovid 
Qui petit seme petit quialt,

et qui auques recoillir vialt, an tel leu sa semance espande que fruit a cent dobles li rande ${ }^{1}$

Chrétien de Troyes

Si l'on en croit le prologue de Cligès, Chrétien de Troyes aurait réécrit plusieurs contes qui trouvent leur origine dans l'Antiquité: Les Commandements d'Ovide, L'Art d'aimer, La Morsure de l'épaule, La Métamorphose de la huppe, de l'hirondelle et du rossignol. II ne parle pas alors de réécriture mais utilise l'expression "en romanz mist". "Mettre en roman", c'est d'abord traduire du latin au roman, au français. Au-delà de la "translation", du passage d'une langue à une autre, l'auteur doit aussi adapter le texte aux préoccupations et aux goûts de son époque. Il convient alors de repérer et d'interpréter la métamorphose culturelle que Chrétien fait subir aux histoires antiques. De plus, il n'a sans doute pas choisi ces œuvres antiques au hasard. Elles soulèvent des problèmes, portent en elles des questionnements qui intéressent l'auteur comme ses contemporains. Pour Chrétien de Troyes, elles sont peut-être aussi le point de départ d'une réflexion qu'il ne cessera de développer tout au long de son œuvre. Ce sont des lectures qui le nourrissent autant qu'il nourrit leur réécriture. Les œuvres citées dans le prologue de Cligès n'ont pas été retrouvées, à l'exception de La Métamorphose de la huppe, présente dans L'Ovide Moralisé et connue désormais sous le titre de Philomena, encore que son attribution ait soulevé de nombreux débats. Nous essaierons de montrer à travers l'exemple de Philomena comment Chrétien opère une transformation, plus qu'une translation, du mythe, le motif de la métamorphose ovidienne devenant un principe d'écriture lui-même porteur de sens à une époque où la naissance du "roman" constitue une étape charnière en littérature.

\section{L'avènement d'un temps nouveau}

Dans le prologue de Cligès, Chrétien se présente dès les premières lignes comme l'auteur de "de la Hupe et de l'Aronde / Et del Rossignol la Muance" que l'on nomme aujourd'hui Philomena. Or, ce prologue souligne la continuité des temps antiques et des temps modernes: "Par les livres que nos avons / Les fez des anciiens savons / Et del siecle qui fu jadis. / Ce nos ont nostre livre apris, / Que Grece ot de chevalerie / le premier los et de clergie. / Puis vint chevalerie a Rome / Et de la clergie la some, / Qui ore est an France

\footnotetext{
${ }^{1}$ Prologue du Conte du Graal: "Qui sème peu, récolte peu, et qui veut avoir belle récole, qu'il jette sa semence en une terre où elle lui rapporte au centuple" (Traduction Jacques Ribard, 1991).
} 
venue." ${ }^{2}$. La transmission des savoirs, qui marque le XII ${ }^{\mathrm{e}}$ siècle, s'illustre dans la réécriture de Philomena où Chrétien de Troyes reprend et adapte pour ses contemporains un texte de l'Antiquité. Ce que les Anciens ont transmis, ils l'ont transmis par les livres. La tapisserie de l'héroïne, qui relate le crime commis par son beau-frère, est donc l'image du livre antique et Philomena apparaît ainsi comme l'histoire d'une double transmission: transmission du récit de la jeune femme à sa sœur et transmission du récit d'Ovide, par Chrétien, à ses contemporains. Toutefois, le prologue se poursuit ainsi: "Mes des Grezois ne des Romains / $\mathrm{Ne}$ dit an mes ne plus mains; / D'aus est la parole remese"3. Cette parole antique évanouie, disparue - car tel est bien le sens de "remese" - n'est-ce pas le silence de Philomena? Des temps anciens à l'époque de Chrétien, il y a à la fois continuité et rupture: continuité par la reprise d'un thème, d'un récit mais rupture de la voix, terriblement matérialisée dans Philomena par cette langue coupée.

D'ailleurs si l'auteur médiéval reprend la temporalité d'Ovide, il la métamorphose aussi. Chez l'auteur latin comme chez Chrétien de Troyes, cinq ans séparent la naissance d'Itys, l'enfant de Térée et Procné, et le moment où cette dernière manifeste le désir de revoir sa sœur. Mais alors que, chez Ovide, l'expression du temps est teintée de mythologie: "Le Titan, ramenant cinq automnes, avait, à autant de reprises déroulé le cours de l'année", elle reste très prosaïque chez Chrétien: "Li anfes crut et amanda / Si fu mout biaus dedanz cinc ans" (v. 42-43) et "Plus de cinc anz esté ansanble / Antre Progné et son seignor" ${ }^{4}$ ( $\mathrm{v}$. 50-51). Concernant le temps de captivité de Philomena, Chrétien en gomme également la référence mythologique et le raccourcit: "Le dieu du jour avait, l'année révolue, parcouru les douze signes." devient "Philomena i fu sis mois" (v. 1144). La métamorphose de ces images n'a pas pour but d'effacer les références païennes puisqu'on sait au contraire que Chrétien n'hésite pas à en ajouter, comme lorsqu'il décrit avec force détails le sacrifice de Procné croyant sa sœur morte. II s'agit plutôt de redonner au temps sa dimension humaine et de souligner le caractère linéaire, au détriment du caractère cyclique, du temps. Or la transmission comme la métamorphose reposent justement sur une conception linéaire du temps.

Chrétien fait subir en outre une autre métamorphose au temps: il semble l'accélérer. Ainsi, lors du voyage qui conduit Térée chez son beau-père et sa belle-sœur, la clémence de la météorologie accélère le temps. De même lors du voyage retour, "Tote est de vant la voile

\footnotetext{
2 "Les livres en notre possession nous ont fait connaître les exploits des Anciens et le monde d'antan, et par nos livres, nous avons appris que la Grèce eut, la première, renom de vaillance chevaleresque et de savoir. Puis la vaillance passa à Rome ainsi que l'ensemble du savoir, qui maintenant est parvenu en France." (Traduction Michel Rousse, 2006).

3 "Des Grecs ou des Romains, on ne dit plus rien désormais. On a cessé de parler d'eux" (Traduction Michel Rousse, 2006).

4 "L'enfant grandit et prospéra, et en cinq ans devint fort beau." et "Déjà Procné et son époux avaient vécu plus de cinq ans ensemble." (Traduction Michel Rousse, 2006).
} 
plainne / Et la nès ne cort mie lant"5 (v. 718-719). Chez Chrétien, plus que les dieux, c'est le temps lui-même, par sa rapidité, sa course inéluctable, qui devient l'image de la fatalité en menant trop vite les personnages vers leur malheur. L'urgence-même des personnages donne l'impression d'accélérer le temps. Si chez Ovide, Térée promet "le prompt retour de la jeune fille en Thrace", chez Chrétien, il donne des délais plus précis dont la brièveté est suspecte "tant solemant trois jorz ou quatre" (v. 515) et qui s'allongent subrepticement "qu'ençois quinzainne / La vos ramanrai" (v. 535-536). Chrétien développe plus qu'Ovide la nuit sans sommeil de Térée, impatient d'emmener Philomena. Le rythme ternaire souligne chez Chrétien la rapidité dont doit faire preuve la fille de la gardienne pour apporter la toile de Philomena à sa sœur: "De revenir ne soies lante. / Va tost et vien sanz demorer." (v. 1226-1227). Enfin, alors que chez Ovide la reine "choisit sa nuit" pour aller rejoindre Philomena, la Procné de Chrétien suit immédiatement la jeune fille qui lui a remis la toile. Tous les personnages paraissent donc plus pressés dans la version médiévale et c'est cette impatience qui les conduit plus rapidement à leur malheur.

Le texte de Chrétien fait apparaître de nombreuses anticipations, comme si l'auteur voulait affirmer sa maîtrise du récit et du temps. Ainsi, à peine a-t-il évoqué la naissance d'Itys au début de son récit qu'il annonce déjà sa mort: "Ce fu diaus granz / Qu'il ne vesqui plus longuemant”' (v. 44-45). A peine Philomena a-t-elle quitté le port qu'il nous révèle qu'elle ne reviendra plus: "S'il an plore mout a grant droit, / Car ja mes ne la reverra"7 (v. 724-725). De manière plus voilée, Chrétien fait comprendre au lecteur que la paysanne chargée de garder Philomena jouera un rôle dans son évasion: "Mes Tereus folie fist / Qui avuec Philomena mist / Por la garder une vilainne"8 (v. 867-869). De telles anticipations apparaissent comme autant de rappels de la fatalité: tout est déjà écrit. Et si l'histoire a déjà été contée par Ovide, le rôle du romancier est d'en infléchir le sens. Avant d'être réécriture, l'adaptation est d'abord relecture.

Dans le traitement du temps, une idée semble obséder Chrétien de Troyes, c'est celle du "bon moment". Dans Philomena, on a l'impression que tout arrive trop tôt, trop vite, ou au contraire trop lentement. Le bateau arrive trop rapidement à Athènes et revient trop rapidement en Thrace et, dès leur arrivée, Térée conduit sa belle-sœur dans une maison isolée et abuse d'elle "sanz nul respit" (v. 795), sans plus tarder. Térée trouve en revanche que son beau-père n'est pas assez prompt à accepter le départ de Philomena, que la nuit qui précède ce départ s'écoule trop lentement ou encore que son fils ne vient pas assez vite les rejoindre, lui et sa femme, alors qu'il est en train de le manger à son insu. Deux

\footnotetext{
5 "Le vent gonfle la voile et le navire file à vive allure" (Traduction Michel Rousse, 2006).

6 "Ce fut une grande douleur que de voir sa vie s'arrêter si tôt" (Traduction Michel Rousse, 2006).

7 "II n'a pas tort de verser des larmes car il ne la reverra plus" (Traduction Michel Rousse, 2006).

8 "Mais Térée commit l'erreur de mettre Philomena en compagnie d'une paysanne qui devait la garder." (Traduction, Michel Rousse, 2006)
} 
proverbes évoquent également cette idée de "bon moment". Le premier se trouve dans la bouche de Térée, lorsqu'il vient annoncer à son épouse la mort de sa sœur: "trop vient a tans / Qui mauveise novele aporte"9 (v. 946-947). Le second proverbe intervient lorsque Philomena veut faire parvenir sans délai sa tapisserie à sa sœur: "Car folie est, dit la letre, / De son afeire respitier / Puisqu'an an puet bien espleitier." ${ }^{\prime 10}$ (v. 1214-1216). Or, le romancier est lui-même confronté à ce problème du "bon moment" dans ses choix d'écriture. A quel moment placer tel épisode? Dans quelle mesure anticiper en révélant des éléments de l'intrigue à venir, surtout si cette intrigue est peut-être déjà connue du lecteur? Faut-il accélérer le rythme du récit ou au contraire le ralentir?

La métamorphose de la temporalité qu'opère Chrétien lorsqu'il reprend le mythe de Philomena, c'est le propre de la "conjointure" qu'évoque notre auteur dans le prologue d'Erec et Enide: "et tret d'un conte d'avanture / une molt bele conjointure"11. Le romancier n'est pas seulement celui qui conte, il est celui qui agence et qui, par cet agencement, redonne du sens.

\section{Métamorphose de la langue}

Pour Chrétien, réécrire Ovide, ce n'est pas seulement faire voyager un récit dans le temps et l'espace, c'est aussi le transposer d'une langue à une autre. Quoi de plus symbolique pour l'auteur des premiers "romans" que de reprendre un mythe latin pour l'adapter en langue romane?

A travers le personnage de Térée, c'est la langue des païens que Chrétien remet en cause. La parole de Térée est mensongère: il trompe son beau-père en lui promettant le retour rapide de sa fille, il ment à sa femme en prétendant que sa sœur est morte. Or, la parole de Térée est explicitement associée au paganisme: "et de ce que je vos promet / Ma foi en ostage vos met / Et tot les deus an qui je croi"12 (v. 537-539). Cette idée est soulignée de nouveau par Philomena qui, pendant que son beau-frère essaie d'abuser d'elle, lui rappelle qu'il a violé le serment fait à son père: "Ou sont li deu? Ou est la foiz?"13 (v. 822). Comble de la violence, Térée veut rendre impossible toute parole de vérité et c'est pour cela qu'il coupe la langue à Philomena ${ }^{14}$.

Dès lors, l'héroïne ne peut plus s'exprimer par la parole et c'est au moyen d'une tapisserie qu'elle apprendra la vérité à sa sœur. La tapisserie est ici métaphore du texte,

\footnotetext{
9 "On arrive toujours trop tôt quand on apporte de mauvaises nouvelles" (traduction Michel Rousse, 2006).

10 "Car il faut être fou, dit le proverbe, pour remettre à plus tard une affaire que l'on peut mener à bien." (Traduction Michel Rousse, 2006).

${ }_{11}$ Ce que René Louis traduit par "il tire d'un conte d'aventure une histoire bien ordonnée".

12 "et pour garants de ma promesse, je vous offre ma parole et tous les dieux en qui je crois" (Traduction Michel Rousse, 2006).

13 "Où sont ces dieux? Où est ta parole" (Traduction Michel Rousse, 2006).

${ }^{14}$ Sur ce thème du mensonge, voir aussi l'article de Waghi Azzam: "La translation dans Philomena".
} 
l'ouvrage est œuvre textuelle. D'ailleurs Chrétien emploie le verbe "écrire": "Tot ot escrit en la cortine" (v. 1131) même si Michel Rousse préfère le traduire par le verbe "décrire": "Tout était décrit sur la tapisserie". En brossant le portrait de Philomena, Chrétien avait déjà souligné son talent pour la broderie qu'il faisait immédiatement suivre de son habileté à "feire vers et letre" (v. 195), comme si finalement ces deux savoir-faire n'étaient pas si éloignés l'un de l'autre. L'écrit, comme l'Ecriture chrétienne, est porteur de vérité et se révèle ainsi supérieur à la parole et à la parole païenne en particulier.

Chrétien précise en outre que la tapisserie de Philomena porte la signature de la jeune femme: "Car tissu ot a l'un des chiés / Que Philomena l'avoit faite"15 (v. 1120-1121). Ce détail n'est pas anodin: alors que le nom de l'auteur est assez secondaire dans l'esprit du public, Chrétien, comme Philomena, signe ses œuvres, inscrit son nom au cœur de ses romans, revendiquant sa part de création personnelle, tant sur le plan de la langue que sur celui de la "conjointure". Dans Philomena, la signature pour le moins obscure, "Crestiiens li Gois", apparaît en plein milieu de l'œuvre, au vers 734, au moment où l'auteur explique que la maison dans laquelle Térée retient prisonnière Philomena se trouve au milieu d'un bois.

Toutefois, la langue de Philomena n'est pas faite de mots mais d'images. Or, ces images ne sont pas comprises par tous. Ainsi, la gardienne de Philomena accepte de remettre la tapisserie à la reine car elle n'arrive pas à comprendre, à interpréter les signes qu'elle voit, ce que Chrétien souligne davantage qu'Ovide: "Mes el ne conut ne ne sot / Rien de quanque cele tissoit, Mes l'uevre li abelissoit"16 (v. 116-1117). La paysanne voit les images, les admire mais elle ne sait pas les lire. En revanche, Procné comprend immédiatement le message de sa sœur. Chez Ovide, elle le comprenait aussi tout de suite mais l'auteur latin s'en étonnait: "mirum potuisse" (v. 583). Au-delà de la "semblance", Procné a su voir la "senefiance". Or, la langue romane, telle que la défend (et l'illustre) Chrétien, ce n'est pas une langue "vulgaire", populaire, mais un langage imagé, porteur de sens et qui demande à être déchiffré.

Le thème de la métamorphose est idéal pour illustrer cette vision de la langue. En effet, la métamorphose est le passage d'une "semblance" à une autre. Elle porte en elle une leçon (que l'Ovide moralisé s'est d'ailleurs efforcé de souligner) et le sens de la métamorphose n'est pas évident, a priori, il demande à être élucidé. Chrétien est plus précis qu'Ovide concernant la métamorphose des deux sœurs: l'auteur latin ne nomme pas explicitement les deux femmes, pas plus qu'il ne nomme les oiseaux. Pour les sœurs, il utilise à deux reprises, le pronom "altera": "quarum petit altera silvas"17 (v. 668), il n'est alors

\footnotetext{
15 "A l'un des bouts, on lisait, inscrit dans le tissage, que c'était l'œuvre de Philomena" (Traduction Michel Rousse, 2006).

16 "mais elle ne comprit rien à ce que la jeune fille tissait; pourtant l'ouvrage lui plaisait" (Traduction Michel Rousse, 2006).

17 "L'une d'elle gagne les forêts" (Traduction Joseph Chamonard, 1966).
} 
nullement question de rossignol; "altera tecta subit, neque adhuc de pectore caedis / excessere notae, signataque sanguine pluma est"18 (v. 669-670): le lecteur devine qu'il s'agit de Procné car c'est elle qui a tué Itys et doit aussi deviner que l'oiseau à la gorge rousse est une hirondelle. Chez Chrétien, "Progné devint une arondele, / Et Philomena rossignos." (v. 1452-1453). On remarque qu'il occulte complètement le crime de Procné. II déplace au contraire la vengeance sanguinaire du côté de Philomena le rossignol dont il interprète le chant: les "Oci! Oci!" qu'elle lance dans la forêt sont autant d'appels au meurtre. Mais ce jeu de mots n'est possible que dans la langue de Chrétien, comme si une langue nouvelle conduisait nécessairement à une interprétation nouvelle du mythe.

Philomena passe successivement de la parole au silence, du silence aux images et des images au chant, à un chant qui doit être compris. Tout se passe donc comme si le chant était à la fois une synthèse et un dépassement du mot et de l'image, ne pouvant être atteint qu'après avoir fait l'expérience du silence. Chrétien fait de cette métamorphose une sorte d'art poétique: l'artiste est celui qui s'empare de la langue vulgaire et qui, par le biais des images, conduit le lecteur au-delà des apparences.

\section{Le temps de la courtoisie}

Le roman de Chrétien ne marque pas seulement l'avènement d'une nouvelle langue littéraire, il ouvre aussi la voie à une nouvelle culture. Tout comme Térée représente la langue des païens, volontiers séductrice et trompeuse, il est aussi le modèle de l'anticourtoisie auquel s'oppose Philomena. Tout d'abord, c'est un être violent: il séquestre sa belle-sœur, la viole ${ }^{19}$, lui coupe la langue. Tous ces actes prennent place au milieu d'une forêt, lieu par excellence de la sauvagerie:

\section{La meisons estoit an un bois, $[\ldots]$ \\ Loing de villes de totes parz \\ Et loing de chans et loing d'essarz, \\ Loing de chemins et de santiers. ${ }^{20}$}

Chrétien reprendra d'ailleurs ce motif de la forêt dans Yvain, le chevalier au lion: le héros, repoussé par sa dame, sombre dans la folie dans une forêt où il vit comme un animal

\footnotetext{
18 "L'autre pénètre sous les toits, et, sur sa poitrine, les marques du meurtre ne se sont pas effacées; son plumage est taché de sang." (Traduction Joseph Chamonard, 1966).

${ }^{19}$ Sur le viol dans Philomena, voir l'article de Michèle Gally: "La raison d'amour sous l'ombre portée du viol".

20 "La demeure était au milieu d'un bois [...], à l'écart des villages de tous les côtés, loin des champs et des cultures, loin aussi de tout chemin et de tout sentier." (Traduction Michel Rousse, 2006).
} 
sauvage $^{21}$. La violence de Térée est perceptible également dans la réaction de ses compagnons qui n'ignorent rien de ce qu'il a fait à Philomena mais se taisent par crainte. Notons qu'Ovide ne mentionnait pas les compagnons. Térée manque aussi de courtoisie dans le choix de son amour: non seulement, il est un homme marié ${ }^{22}$ mais il désire commettre un adultère avec la sœur de son épouse. Cet amour a quelque chose d'incestueux, bafouant les liens familiaux, cela explique peut-être que, pour se venger, Procné brave, toujours au sein de la famille, un autre interdit: infanticide et anthropophagie ont pour victime leur propre enfant. Pourtant, Térée, chez Chrétien, se montre capable d'une réaction courtoise: il essaie d'obtenir l'amour de Philomena avant d'abuser d'elle.

\section{Mes de tant fet viaus que cortois}

Que s'amor li requiert ençois

Qu'il li forface nule rien ${ }^{23}$. (v. 763-765)

Mais là encore, la parole de Térée manque de courtoisie car lorsque Philomena refuse de l'aimer comme il le désire, il ne tient pas compte de sa réponse, se montre de plus en plus violent. Sa courtoisie était donc toute rhétorique.

Face à Térée, Philomena est la dame courtoise par excellence. L'énumération de ses qualités et de ses talents par Chrétien semble bien peu s'appliquer à un personnage de l'Antiquité: elle pratique des jeux à la mode au Moyen-Age, maîtrise l'art de la fauconnerie, joue de nombreux instruments dont la vielle... Alors qu'Ovide ne mentionnait que sa beauté, sa parure et la grâce de sa démarche, Chrétien rappelle que l'idéal du Moyen-Age allie perfection physique et morale: "Nu fu pas mains sage que bele" (v. 172).

Comme c'est le cas traditionnellement avec la fin'amor, c'est bien la dame qui éduque, ou du moins tente d'éduquer, l'homme aux valeurs courtoises. Lorsque Térée demande à sa belle-sœur d'intervenir directement pour convaincre son père de la laisser partir avec lui, Philomena, dans un anachronisme extraordinaire, invoque "la costume as François", la coutume des Français. Elle refuse d'intervenir et dit que c'est à lui de convaincre le vieux roi car c'est lui qui veut son départ. Puis les deux personnages interprètent de manière contradictoire le silence du vieux Pandion face à la demande de Térée: pour l'une, c'est un refus; pour l'autre, c'est un accord tacite. Dans le bois, Térée et Philomena s'opposent sur la définition de l'amour: lui aime sa belle-sœur d'un amour déshonnête qui doit rester secret alors qu'elle avoue sans ambages un amour quasi fraternel: "Je vos aim bien si con je doi" (v. 772). Mais Térée refuse d'entendre le langage de

\footnotetext{
${ }^{21}$ Sur le motif de la forêt dans l'œuvre de Chrétien de Troyes, voir mon article "Le chêne et le charme: la forêt hors les lois dans l'œuvre de Chrétien de Troyes".

${ }_{22}$ Or, dans Erec et Enide, Chrétien de Troyes fait bien l'apologie d'un amour courtois au sein du mariage.

23 "Pourtant, un reste de courtoisie le pousse à requérir d'amour la jeune fille plutôt que de la brutaliser" (Traduction Michel Rousse, 2006).
} 
Philomena, il ne veut pas être éduqué à la courtoisie et son geste est extrême: il lui ôte la parole.

La fin du texte de Chrétien souligne de nouveau le rôle de Philomena dans l'éducation à la courtoisie. Le rossignol qu'elle est devenue condamne par son chant les hommes dont le comportement va à l'encontre de la courtoisie:

\author{
Ancore, qui crerroit son los, \\ Seroient à honte trestuit \\ Li desleal mort et destruit \\ Et li felon et li parjure \\ Et cil qui de joie n'ont cure \\ Et tuit cil qui font mesprison \\ Et felenie et traïson \\ Vers pucele sage et cortoise (v. 1454-1461) ${ }^{24}$
}

Le dialogue de Térée et Philomena est redoublé par le dialogue fictif entre le lecteur et le narrateur qui, comme souvent dans les romans courtois, se livrent à une réflexion sur les sentiments de Térée et ce qui le pousse à agir. L'accès à la courtoisie apparaissant ainsi comme profondément dialogique, le silence imposé à Philomena fait figure d'aporie.

Par rapport à Ovide, Chrétien développe grandement la parole des personnages et particulièrement celle de Térée ${ }^{25}$. Dans le dialogue entre Térée et Philomena, le lecteur entend le dialogue de deux cultures: au sein d'un mythe païen où se déchaînent les désirs non maîtrisés et la violence, la voix de Philomena annonce le roman courtois. Et c'est bien sa voix qui clôt le roman. Lorsque Chrétien écrit: "De Philomena leirai ci"26, il s'agit moins d'une conclusion que d'une transition: le conte Philomena laisse place à des romans libérés du joug antique, où la courtoisie pourra s'exprimer librement.

Même si Chrétien fait une plus large place au dialogue, il accorde un statut tout particulier au silence, valeur courtoise à part entière dans son œuvre. Le silence, avec le motif de la langue coupée, est inscrit au centre du conte. Mais Chrétien estompe le caractère spectaculaire qu'Ovide conférait à ce motif:

radix micat ultima linguae,

ipsa iacet terraeque tremens inmurmurat atrae,

utque salire solet mutilatae cauda colubrae,

\footnotetext{
24 “Aujourd'hui encore, si l'on suivait le conseil de Philomena, tous les traîtres seraient voués à une mort honteuse, ainsi que les scélérats et les parjures et ceux qui ne font aucun cas de la joie, ou tous ceux qui commettent d'ignobles forfaits en trahissant des jeunes filles sages et courtoises." (Traduction Michel Rousse, 2006).

${ }^{25}$ La parole de Térée n'occupe qu'un seul vers dans la version d'Ovide!

26 "Je terminerai ici le conte de Philomena." (Traduction Michel Rousse, 2006).
} 
palpitat et moriens dominae uestigia quaerit. ${ }^{27}$ (v. 557-560).

En revanche, il étoffe le thème du silence qui parcourt désormais plus largement le conte. Le silence est d'abord celui de Pandion. Lorsque Térée lui demande la permission de conduire Philomena chez sa sœur, le vieux roi ne répond pas. Dès que s'achèvent les paroles de Térée, qui se plaint d'ailleurs de ne pas avoir vu sa belle-sœur, cette dernière apparaît. Elle est tellement éblouissante et la liste de ses qualités tant physiques que morales est si longue que tout le monde, y compris le narrateur, semble avoir oublié la prière de Térée. Ce dernier reprend ensuite la parole pour demander à Philomena de convaincre son père de la laisser partir. S'ensuit alors une discussion sur le silence de Pandion. Le silence est glosé: il demande à être interprété. Plus tard, dans la forêt, Térée demande à Philomena de taire un amour illicite, de le cacher à tous alors que Philomena demande à Térée de ne plus lui parler de ce sentiment, de le faire disparaître. Leur interprétation du silence diverge encore, comme face au silence de Pandion. Pour Térée, le silence est un accord tacite: Philomena doit accepter sans un mot ses avances. Pour Philomena, le silence est un refus. II y a sans doute un jeu de mots entre le verbe "teire" et le nom du personnage, Térée: "Teirai?" demande-t-il incrédule à Philomena au vers 777 . Térée est en effet celui qui ne peut pas se taire et qui ne parvient pas non plus à faire taire ses bas instincts. Chrétien, prêtant cet échange sur le silence aux personnages, souligne l'ironie tragique: Philomena demande à son beau-frère de se taire mais c'est elle qui perd la parole. Pourtant Térée est aussi celui qui ne parvient pas à obliger les autres à se taire, Philomena trouvant un autre moyen que la parole pour faire parvenir son message à sa sœur.

Le silence, c'est encore celui des compagnons de Térée, n'ignorant pourtant pas le crime. C'est celui de Procné, qui comprenant le sens de la tapisserie, retient ses cris. C'est celui de la paysanne qui garde Philomena lorsque Procné frappe à la porte de la maison au milieu du bois. C'est enfin celui d'Itys qui demeure tragiquement muet, se contentant d'enlacer et d'embrasser sa mère avant que celle-ci ne le tue. Chez Ovide, on l'entendait au moins s'exclamer "mater! mater!" (v. 640).

Dans Le Conte du Graal, Chrétien réaffirmera les vertus du silence, avec un roman inachevé qui se termine donc lui-même par le silence de l'auteur. Le jeune Perceval pose trop de questions, parle à tort et à travers, n'écoute pas suffisamment les autres. Ainsi, dans l'enseignement qu'il lui dispense, Gornemant le met en garde contre une parole abusive:

et li saiges dit et retrait:

"Qui trop parle pechié fet."

\footnotetext{
27 "La racine en palpite au fond de la bouche; la langue elle-même, jetée sur le sol, murmure ses plaintes à la terre qu'elle noircit de son sang. Comme on voit sursauter la queue coupée d'une couleuvre, elle palpite et, avant de mourir, cherche à rejoindre celle à qui elle appartient." (Traduction Joseph Chamonard, 1966).
} 
Por ce, biau frere, vos chasti

de trop parler,[...] (v. 1651-1654) ${ }^{28}$

On sait toutefois que le conte ne prône pas un silence absolu. En effet, si Perceval n'a pas accès aux mystères du Graal, c'est que, suivant trop à la lettre les recommandations de Gornemant, il ne pose aucune question sur le Graal.

Au-delà du thème du silence, ou plutôt de celui d'une parole maîtrisée, réfléchie, plusieurs thèmes sont en germe dans Philomena, qui seront repris dans d'autres œuvres. Nous nous intéresserons au cas du Conte du Graal. Le bois isolé dans lequel Térée retient Philomena prisonnière n'est pas sans rappeler la "gaste forest" dans laquelle Perceval et sa mère vivent reclus. Philomena n'est en contact qu'avec la paysanne qui la garde; de la même manière, Perceval ne côtoie que les paysans qui travaillent pour sa mère, cette dernière tenant à éloigner son fils du monde de la chevalerie afin qu'il ne meure pas au combat comme son père et ses frères. Comme dans Philomena, il est aussi question de viol dans Le Conte du Graal. Lorsque Perceval rencontre pour la première fois une jeune fille, il pénètre dans sa tente en l'absence de son ami et, malgré son interdiction, lui vole des pâtés et la bague qu'elle portait. Mais, après une initiation à la courtoisie, il comprendra sa faute et saura la racheter. Si Philomena est l'histoire d'un infanticide (Procné tue son fils pour se venger de son époux), Le Conte du Graal est l'histoire d'un matricide. Lorsque Perceval découvre la chevalerie, il quitte sa mère. Lors de son départ, il se retourne et voit qu'elle "jut pasmee an tel meniere / com s'ele fust cheüe morte"29 (v. 622-623). II ne fait pas demi-tour mais cingle au contraire la croupe de son cheval pour partir plus vite. II apprendra dans la suite du roman que sa mère est morte. Soit Perceval mourait en quelque sorte étouffé par sa mère, soit il la tuait. Le mythe d'Ovide met en scène, de manière implicite, la rivalité entre les deux sœurs. Même si elles se soutiennent et se liguent dans le crime, elles ont toutes deux partagé le même homme. Dans Le Conte du Graal, Chrétien montre plus clairement, dans un épisode qui a pour héros Gauvain, la rivalité entre deux sœurs. L'aînée a giflé la cadette qui demande réparation auprès de Gauvain $^{30}$. Le chevalier courtois doit donc régler les conflits entre les sœurs, non risquer de les allumer comme le fait Térée. Si l'on compare les descriptions de Philomena et Blanchefleur, la femme aimée par Perceval, on constate qu'elles se ressemblent étrangement, ce qui n'est guère étonnant, le portrait féminin étant un

\footnotetext{
28 "Comme le dit si bien le proverbe: "A trop parler, faute on commet." Voilà pourquoi, mon ami, je vous recommande de ne pas trop parler" (Traduction Jacques Ribard, 1991).

29 "Elle était étendue sans connaissance, comme morte." (Traduction Jacques Ribard, 1991).

${ }^{30}$ II y a un épisode similaire dans Le chevalier au lion où deux sœurs se disputent l'héritage. Gauvain défend l'une et Yvain, sous le nom du chevalier au lion, défend l'autre.
} 
passage obligé assez stéréotypé ${ }^{31}$. Leurs cheveux sont comparés à de l'or fin, elles ont le front "blanc" et "plain", c'est-à-dire lisse, les sourcils écartés ${ }^{32}$, le nez "droit". La description physique de Philomena est toutefois plus développée, Chrétien évoquant les seins, les mains, la taille et les hanches, ce qu'il ne fait pas pour Blanchefleur. Pourtant, on peut dire que c'est le portrait de Blanchefleur qui est le plus abouti. En effet, si le désir de Térée est éveillé et soutenu par la vue de Philomena, l'amour de Perceval est entretenu par le souvenir de son amie. II s'absorbe ainsi dans la contemplation de gouttes de sang sur la neige qui lui rappellent les couleurs du visage de Blanchefleur ${ }^{33}$. On remarque à cette occasion que le sang, lié à la violence dans Philomena, atteint ici une dimension symbolique, tout comme les gouttes de sang qui perlent de la lance qui accompagne le cortège du graal. Autre évolution dans le portrait: la beauté de Philomena est l'œuvre de Dieu et de la Nature

\author{
Tel l'ot Dieu feite que Nature \\ Mien esciant i fausist bien \\ S'ele i vosist amender rien (v. 142-144) \\ Car Nature s'en fu penée \\ Plus que de nule autre bien née, \\ S'i ot tot mis quanqu'ele pot. (v. 167-169) ${ }^{34}$
}

alors que la beauté de Blanchefleur est l'œuvre de Dieu seul:

fist Dex de li passemervoille, n'onques puis ne fiste la paroille ne devant faite ne l'avoit. (v. 1825-1827) ${ }^{35}$.

Enfin, Philomena peut apparaître à certains égards comme la préfiguration du cortège du graal. Lorsqu'elle apparaît, Chrétien la décrit ainsi "qui la face ot vermoille et clere" (v. 206). Les couleurs de son teint rappellent celles de la lance qui accompagne le graal: blanche avec des gouttes de sang. L'adjectif "clere" évoque aussi la lumière, lumière très présente également lors du passage du cortège du Graal. Lorsque la demoiselle qui porte le graal entre dans la pièce, l'éclat du graal surpasse alors la lumière des chandeliers.

\footnotetext{
${ }^{31}$ Voir à ce propos la comparaison des portraits des héroïnes de Chrétien de Troyes dans l'article de Carmelle Mira: "Un topos médiéval, le chef d'œuvre inimitable... et sans cesse imité: la beauté de l'héroïne chez Chrétien de Troyes."

${ }^{32}$ Chrétien de Troyes emploie l'expression "large antr'oel" (v. 145 de Philomena et v. 1817 du Conte du Graal.)

${ }^{33}$ De la même façon, Lancelot dans Le chevalier de la charrette, se plonge dans la contemplation d'un peigne ayant appartenu à Guenièvre.

34 "C'était l'œuvre de Dieu lui-même, et Nature, à mon avis, aurait échoué à faire mieux. [...] Nature avait mis plus de soin à la faire que pour aucune autre créature; elle y avait employé toutes ses ressources." (Traduction Michel Rousse, 2006).

35 "Dieu avait fait d'elle la merveille des merveilles; il n'en avait jamais fait de semblable et n'en fit jamais plus." (Traduction Jacques Ribard, 1991).
} 
Un ermite expliquera plus tard à Perceval que le graal contient une hostie qui permet à elle seule de maintenir en vie le vieux père du roi Pêcheur. Or, Pandion affirme que Philomena lui est indispensable, vitale même: "Que ce ne fust ses reconforz, / grant pieç'a que je fusse morz"36 (v. 377-378). Son grand âge en fait d'ailleurs un personnage surnaturel, à l'image du père du roi Pêcheur: "Plus ai vescu ne fist Jacob / Ne Abraham"37 (v. 360-361).

Tout se passe donc comme si Philomena contenait déjà en germe nombre d'éléments qui trouveront leur aboutissement dans Le Conte du Graal, comme si Chrétien semait déjà dans la réécriture du mythe antique les idées qui devaient s'épanouir dans son dernier roman. Dans le prologue du Conte du Graal, Chrétien de Troyes compare d'ailleurs le comte Philippe de Flandre à Alexandre:

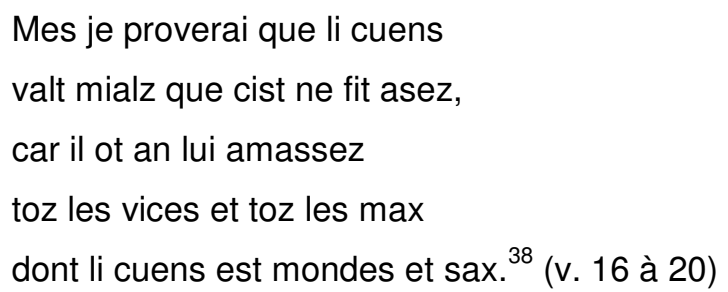

De Philomena au Conte du Graal, on assiste à une purification des mœurs, à une christianisation de la pensée qu'illustrent bien les deux scènes de repas qui sont d'une part le festin anthropophage de Térée dévorant son propre fils, d'autre part l'ascèse du repas du graal.

Ce n'est pas un hasard si Philomena se clôt sur le motif de la reverdie. "Quant il vient au prin d'esté" (v. 1463), le rossignol qu'est devenue l'héroïne se met à chanter dans les bois. Habituellement, la reverdie ouvre les romans, comme c'est le cas notamment du Conte du Graal: "Ce fu au tans qu'arbre florissent, / feuillent boschaige, pré verdissent" ${ }^{39}$ (v. 69-70). Mais la fin de Philomena est bien une ouverture, une ouverture sur le roman qui véhicule les valeurs courtoises et chrétiennes. II existe une véritable continuité dans l'œuvre de Chrétien de Troyes, comme il existe une continuité évidente, pour l'auteur médiéval, entre la littérature des anciens et celle de son époque. En reprenant ce texte d'Ovide, Chrétien illustre le fait que la "translatio" est métamorphose: elle transmet en modifiant la forme et le sens. La fin de Philomena qui invite à entendre "ancore" (v. 1454) le chant de l'héroïne montre que le mythe traverse les époques mais le message que contient le chant et donc le mythe se transforme au fil du temps.

\footnotetext{
36 "Sans le réconfort qu'elle m'apporte, il y a longtemps que je serais mort." (Traduction Michel Rousse, 2006).

${ }^{37}$ Selon la Bible, Jacob aurait vécu jusqu'à 147 ans et Abraham jusqu'à 175 ans.

38 "Et je montrerai que le comte lui est bien supérieur, car Alexandre portait en lui tous les vices et toutes les faiblesses dont le comte s'est purifié et libéré." (Traduction Jacques Ribard, 1991).

39 "C'était au temps où les arbres fleurissent, où les bois se couvrent de feuilles et les prés de verdure" (Traduction Jacques Ribard, 1991).
} 


\section{Bibliographie}

AzZAM, Wagih (1989), "Le printemps de la littérature. La 'translation' dans Philomena de Crestiiens li gois." [en ligne]. In: Littérature, $N{ }^{\circ} 74$, Le miroir et la lettre. Écrire au Moyen Âge. pp. 47-62. [consulté le 09/07/2012]

<URL: http://www.persee.fr/web/revues/home/prescript/article/litt_0047-

4800_1989_num_74_2_1482>.

CARRETO, Carlos, Figuras do silêncio: di inter-dito a emergência da palavra no texto medieval, Lisboa: Estampa.

Chrétien de Troyes (2006), Cligès, Philomena, traduction et présentation par Michel Rousse, Paris: GF Flammarion.

(1984), Le Conte du Graal, tomes 1 et 2, publié par Felix Lecoy, Paris:Honoré Champion.

(1991), Le Conte du Graal, traduit par Jacques Ribard, Paris: Honoré Champion traduction.

GALLY, Michèle (2003), "La raison d'amour sous l'ombre portée du viol". In: Jean-René Vallette, Jean-Claude Vallecalle, Marie-Etiennette Bély (coll.) Entre l'ange et la bête: l'homme et ses limites au Moyen-Age [Actes des deux colloques organisés à Lyon en avril 2000 et avril 2001, Presses Universitaires de Lyon, pp. 187-195.

GINGRAS, Francis (2004), "Aimer hors chant: réinvention de l'amour et invention du 'roman"” [en ligne]. In: Intermédialités: histoire et théorie des arts, des lettres et des techniques / Intermediality: History and Theory of the Arts, Literature and Technologies, $\mathrm{n}^{\circ} 4$, pp. 18-43. [consulté le 11/07/2012] <URL: http://id.erudit.org/iderudit/1005475ar>.

JAMES-RAOUL, Danièle (2000), La parole empêchée dans la littérature arthurienne, Paris: Honoré Champion.

MIRA, Carmelle (1999), "Un topos médiéval, le chef d'œuvre inimitable... et sans cesse imité: la beauté de l'héroïne chez Chrétien de Troyes" [en ligne]. In Arob@se, Journal des lettres \& sciences humaines, Volume 3, n 2. [consulté le 18/11/2012] <URL: http://www.univrouen.fr/arobase/bck6.html>.

OVIDE (1960), Les Métamorphoses, VI "Progné et Philomèle", traduction et notes par Joseph Chamonard, Paris: GF Flammarion, pp. 167-174.

RoustanT, Sylvia (2010), "Le chêne et le charme: la forêt hors-les-lois dans l'œuvre de Chrétien de Troyes". In: Otrante, art et littérature fantastiques, n`27-28 "Forêts fantastiques" collectif sous la direction de Lambert Barthélémy, Paris, Editions Kimé, pp. 49-58. 